The Astrophysical JournaL, 162:475-483, November 1970

(C) 1970 The University of Chicago All rights reserved Printed in U.S A.

\title{
OPTICAL POLARIZATION AND INTENSITY OF THE PULSAR IN THE CRAB NEBULA
}

\author{
J. KRISTIAN \\ Hale Observatories, Carnegie Institution of Washington, California Institute of Technology \\ N. Visvanathan \\ Harvard College Observatory \\ AND \\ J. A. Westphal and G. H. Snellen* \\ Hale Observatories, Carnegie Institution of Washington, California Institute of Technology \\ Received 1970 June 23
}

\section{ABSTRACT}

Broad-band photoelectric measurements on nine nights between 1969 February and 1970 February show constant intensity and colors for the pulsar of $V=16.5, B-V=+0.5, U-B=-0.45$, and $V-R=-0.75$, with an accuracy of 10-15 percent in each passband. The ratio of the intensity in the main pulse to that in the subpulse is about 1.8 in each color, with the same accuracy. In the passband $\lambda \lambda 3800-6000$, the pulsar intensity repeated within 3 percent on nights 2 months apart.

Measurements of linear polarization on four nights in 1969 February, August, and October show repeatable behavior, with the amount of polarization changing smoothly through the pulse from at least 25 percent to near zero and the plane of polarization sweeping through $150^{\circ}$ during $60^{\circ}$ of the pulsar's rotation. The time behavior was similar in the main pulse and the subpulse. The discontinuous change of the pulsar's period in 1969 September had no discernible effect upon the intensity, colors, or polarization of the pulses. It is argued that the observed pulses are due to a fixed, polarized emission pattern which is azimuthally scanned as the object rotates, and the sweep of the plane of polarization is interpreted in terms of a very general geometrical model. If the model is valid, then the projection of the rotation axis of the pulsar on the plane of the sky is found directly from the data, with an ambiguity of $90^{\circ}$, and is either parallel or orthogonal to the integrated magnetic field of the nebula within $20^{\prime \prime}$ of the pulsar. This agreement between the angles of the rotation axis and the magnetic field of the nebula seems too close to be accidental, and implies that the external field, at least in the vicinity of the pulsar, was produced by the pulsar at some stage in its history.

\section{INTRODUCTION}

We measured the linear polarization and broad-band intensities of the optical pulsar NP 0531 in the Crab Nebula several times between 1969 February and 1970 February. The measurements were made at the prime focus of the Hale 200-inch telescope with a 1P21 photomultiplier and at the Newtonian focus of the 100 -inch with an S-20 photomultiplier. Most of the data described in this paper were obtained with a Fabri-Tek signal averager, operating in the pulse-counting mode and cycled at a multiple of the pulsar period, with time resolution of either 40 or $50 \mu \mathrm{sec}$. The experiment is essentially that described by Westphal et al. (1969); digital magnetic recording of raw data was used in place of the analog recording used earlier.

\section{BROAD-BAND PHOTOMETRY}

Table 1 shows the time-averaged broad-band intensities of the pulses after subtraction of the background, as measured on nine separate nights between 1969 February and 1970 February. $U B V$ magnitudes are in the Johnson-Morgan system, and $R$ is in the system of Sandage and Smith (1963). The main pulse is defined as the interval from 4

* Now at Massachusetts Institute of Technology. 
TABLE 1

BROAD-BAND PHOTOMETRY OF NP 0531

\begin{tabular}{|c|c|c|c|c|c|c|c|c|}
\hline \multirow[b]{2}{*}{ Date } & \multicolumn{4}{|c|}{$\begin{array}{c}\text { Total Magnitudes } \\
\text { (main pulse + subpulse) }\end{array}$} & \multicolumn{4}{|c|}{$\begin{array}{l}\text { INTENSITY Ratios } \\
\text { (main pulse/subpulse) }\end{array}$} \\
\hline & $V$ & $B$ & $U$ & $R$ & $V$ & $B$ & $U$ & $R$ \\
\hline \multirow{9}{*}{$\begin{array}{l}\text { 200-inch: } \\
1969 \text { Feb. } 10 \ldots \ldots \\
1969 \text { Mar. } 14 \ldots \ldots \\
1969 \text { Aug. } 19 \ldots \ldots \\
1969 \text { Aug. } 20 \ldots \ldots \\
1969 \text { Aug. } 21 \ldots \ldots \\
\text { 100-inch: } \\
1969 \text { Sep. } 17 \ldots \ldots \\
1969 \text { Sept. } 18 \ldots \\
1969 \text { Nov. } 16 \ldots \\
1970 \text { Feb. } 2 \ldots \ldots\end{array}$} & \multirow{5}{*}{$\begin{array}{l}16.5 \pm 0.15 \\
16.5 \pm 0.15 \\
16.6 \pm 0.15 \\
16.3 \pm 0.1 \\
16.5 \pm 0.1\end{array}$} & & & & \multirow{5}{*}{$\begin{array}{l}2.1 \pm 0.3 \\
1.65 \pm 0.15 \\
1.8 \pm 0.15 \\
1.9 \pm 0.25\end{array}$} & & \multirow[b]{2}{*}{$\ldots$} & \multirow{3}{*}{$\cdots$} \\
\hline & & $16.9 \pm 0.15$ & $16.5 \pm 0.15$ & $\ldots$ & & & & \\
\hline & & & & $\ldots$ & & & & \\
\hline & & $17.2 \pm 0.15$ & $16.7 \pm 0.2$ & $\ldots$ & & $1.85 \pm 0.1$ & $2.0 \pm 0.15$ & $\ldots$ \\
\hline & & $16.9 \pm 0.1$ & $16.45 \pm 0.15$ & $\ldots$ & & $1.95 \pm 0.15$ & $2.0 \pm 0.2$ & $\ldots$ \\
\hline & & & & & & & & \\
\hline & $16.6 \pm 0.15$ & & & & $1.3 \pm 0.4$ & & & \\
\hline & $16.7 \pm 0.15$ & $17.25 \pm 0.15$ & $17.0 \pm 0.3$ & $15.8 \pm 0.15$ & $2.0 \pm 0.35$ & $2.0 \pm 0.35$ & $1.8 \pm 0.7$ & $1.8 \pm 0.3$ \\
\hline & $\leq 16.7 \pm 0.2$ & $\begin{array}{r}17.3 \pm 0.2 \\
\leq 17.3\end{array}$ & $\begin{aligned} & 16.8 \pm 0.4 \\
& \leq 16.8\end{aligned}$ & $\begin{array}{r}16.0 \pm 0.2 \\
\leq 16.2\end{array}$ & $\begin{array}{l}1.75 \pm 0.15 \\
1.7 \pm 0.3\end{array}$ & $\begin{array}{l}2.0 \pm 0.15 \\
1.7 \pm 0.3\end{array}$ & $\begin{array}{l}2.0 \pm 0.3 \\
1.45 \pm 0.7\end{array}$ & $\begin{array}{l}1.9 \pm 0.2 \\
1.75 \pm 0.35\end{array}$ \\
\hline Average....... & $16.5 \pm 0.1$ & $17.0 \pm 0.15$ & $16.55 \pm 0.15$ & $15.85 \pm 0.1$ & $1.75 \pm 0.1$ & $1.85 \pm 0.1$ & $1.9 \pm 0.15$ & $1.85 \pm 0.05$ \\
\hline
\end{tabular}


msec preceding the peak to $4 \mathrm{msec}$ following it. The subpulse is defined as the interval from $4.5 \mathrm{msec}$ preceding the peak to $5.5 \mathrm{msec}$ following it. The background in both cases is defined as the interval from 8 to $4 \mathrm{msec}$ preceding the peak of the main pulse. There is evidence that the pulsar is in fact off during this interval, with an uncertainty of a few percent (Miller and Wampler 1969; Kristian 1970).

Table 1 lists (1) total $V_{-}, B_{-}, U_{-}$, and $R$-magnitudes for the sum of the main pulse and the subpulse, averaged over the total integration time, including the time that the pulses are off, and (2) the ratio of the intensity of the main pulse to the intensity of the subpulse for each color. The listed magnitudes are those which would be measured by conventional photometric techniques with integration times long compared with the pulsar period.

On 1970 February 10, during the course of the observations of the pulsar, clouds began to form in a previously stable sky. It is likely that the $V$-magnitude is accurate and that the other measurements are within 20-30 percent of the true values. These measurements were not used in calculating the average magnitudes, but are included to indicate that the pulsar had not changed drastically since the last observation $2 \frac{1}{2}$ months earlier. The ratios are taken to be valid, since they are independent of extinction if the colors of the main and subpulse are the same, and all measurements to date indicate that they are.

To within the error of the measurements $(\sim 15$ percent), the intensities of both the main pulse and the subpulse are constant in each passband from night to night over the 12 months spanned by the observations. More accurate evidence for constancy is obtained from the measurements of polarization discussed in $\$$ III below, which used a wider passband $(\lambda \lambda 3800-6000)$ : the intensities of the pulsar measured on the nights of 1969 August 18, August 19, and October 19 were the same to within 3 percent. It should be noted that these measurements and those of Table 1 span the occurrence of the period "jump" of the pulsar sometime in 1969 September (Boynton et al. 1969).

The errors quoted in Table 1 include both statistical and calibration uncertainties. Within a given night, comparisons of the pulsar at different times will be subject to statistical uncertainties only and will therefore be of higher accuracy. On several nights, repeated measurements were made which spanned total times of the order of an hour. In each case, intensities and colors were constant within the statistical accuracy of 3-5 percent for the main pulse and 5-8 percent for the subpulse. Constancy on these and shorter time scales has been reported earlier in our data (Kristian 1970) and those of Wampler, Scargle, and Miller (1969). Also, our observed pulse shapes are found to be constant in time and in all colors (i.e., the color of the emitted light is constant through the averaged pulses), as previously reported by other groups (Wampler et al. 1969; Warner, Nather, and MacFarlane 1969).

Our intensities agree within the errors with a measurement of $V=16.5, B-V=$ $0.6, U-B=-0.6$ made in 1969 January by Lynds, Maran, and Trumbo (1969). Earlier suggestions of large night-to-night variations in intensity (see, e.g., Cocke, Disney, and Gehrels 1969; Freeman et al. 1969) are not confirmed. Our broad-band colors agree reasonably well with spectral scans obtained by Oke (1969) on the nights of 1969 February 7,8 , and 9 . Also, our $V$-magnitude of 16.5 on February 10 and our total average $V$-magnitude of 16.5 agree with Oke's $V$-magnitudes of 16.5 and 16.6 on February 7 and 9 , but disagree substantially with his magnitude of 16.1 on February 8 . If the latter value is accurate, it is a major anomaly in an otherwise homogeneous series of results. Oke's experiment, however, was aimed primarily at determining the energy distribution of the pulsar, rather than its absolute flux, and the apparent discrepancy should probably not be given high weight (Oke, private communication). It should be noted that the energy distribution published by Oke (his Fig. 1) has been normalized to $V=16.1$.

\section{POLARIZATION}

Linear polarization of the pulsar was measured on the nights of 1969 February 8, August 18, August 19, and October 19, at the prime focus of the 200-inch, with the singlechannel polarimeter described by Sandage and Visvanathan (1969). In February, mea- 
surements were obtained by digitally recording raw photon counts for successive 1-msec integration times, then later averaging the recorded raw data by computer. The starting time of each run was not known sufficiently accurately to compare the phase from angle to angle, so that for this run, the data gave direct information only on the polarization of the nebula and the integrated pulses. The August and October measurements were made by synchronously averaging the photomultiplier counts at the telescope for 1 or 2 minutes at each setting of the Polaroid angle. The starting time of each run was known accurately enough that the detailed time behavior of the polarization within the averaged pulses could be studied. Measurements were made with the Polaroid angle at $30^{\circ}$ intervals, and the data were reduced in the normal way by fitting to a double sine curve (Visvanathan 1967, 1968).

\section{a) Polarization of the Nebula near the Pulsar}

In those parts of the light curve of the pulsar between pulses, where the pulsar itself is turned off, the data give the polarization of that part of the nebula which is within the focal-plane diaphragm. The polarization $P$ is found to be $12.2 \pm 0.2$ percent at an angle $\theta$ of $159^{\circ} \pm 2^{\circ}$ (measured north through east), and is the same for aperture diameters of $5^{\prime \prime}, 12^{\prime \prime}$, and $19^{\prime \prime}$. These numbers agree well with several measurements of the nebula within $20^{\prime \prime}$ of the pulsar, made with a 6" aperture (Visvanathan 1970), and with a previous measurement by Hiltner (1957) at a point in the nebula about $20^{\prime \prime}$ from the pulsar, with an aperture of $5^{\prime \prime}$. These facts indicate that the average magnetic field around the pulsar is uniform over distances of the order of $0.02-0.2 \mathrm{pc}$ from the pulsar. Furthermore, the agreement between the polarization at the minima of the pulsar light curve and the polarization of the nearby nebula may be taken as additional evidence that the pulsar is effectively turned off between pulses.

The plane of polarization of the nebula is not oriented in a simple way with respect to the direction of the structure visible on continuum photographs. Published plates by Baade (1956) and Münch (Scargle 1969) show considerable structure in the nearby nebula, all at a position angle of about $35^{\circ}$ (note that the position angle of the central pair of stars is 31.5 [Seeger and Westerhout 1957]). Our measurements show that the integrated electric vector forms an angle of about $55^{\circ}$ with respect to this structure, so that the visible structure does not appear to be aligned with the integrated magnetic field. Measurements of the nebula by Visvanathan (1970) show a discontinuity of $\theta$ at Scargle's Wisp 2, confirming that the field within the wisps is not parallel to the largerscale field of the nearby nebula.

\section{b) Polarization of the Integrated Pulses}

For each Polaroid angle, the total net intensity of the pulses was measured and was then used to compute a polarization formally in the usual way. As we will shortly see, the polarization changes drastically within the pulse, so that this integrated polarization has no direct physical meaning. It is useful, however, as an index, of relatively high accuracy, to the gross properties of the polarization of the pulsar at a given epoch, and has been compared with measurements by other observers (cf. § III $e$ below).

Figure 1 shows the integrated polarization of the main pulse for each of the three months. The accuracy of the fit of each set of data to the theoretical curve shows that for each night there are no large secular changes of gross polarization or intensity of the pulsar over periods of about an hour, the total time spanned by each run. Further, the agreement of the separate sets of data with one another shows that there are no changes in the gross polarization properties of the pulsar over the 8 months spanned by the observations.

\section{c) Change of Polarization within the Pulses}

The polarization was computed individually for each observed point within the pulses for the data of August and October, after subtraction of the background. Figures 2 and 3 


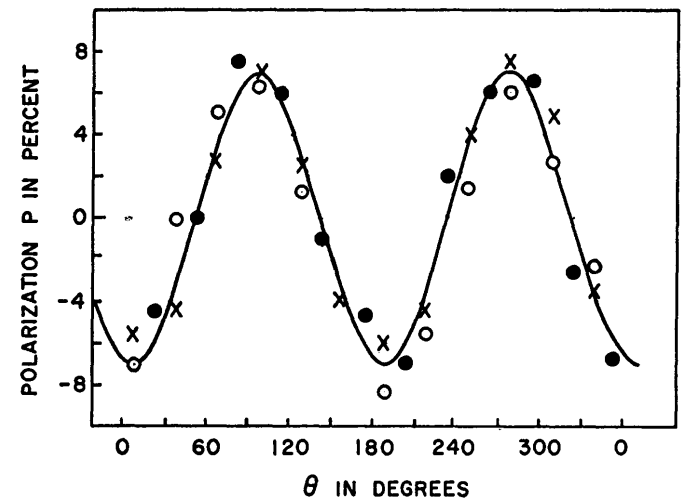

Fig. 1.- Integrated linear polarization of the main pulse. The integrated polarization is a formal quantity computed from the integrated pulse intensities at the indicated Polaroid angles (cf. $\S \mathrm{III} b$ ). Data were taken on 1969 February 8 (crosses), August 18 and 19 (average of 2 nights, open circles) and October 19 (filled circles). The best fit to a double sine curve gives a polarization $P=6.8 \pm 0.5$ percent with the electric vector at $\theta=98^{\circ} \pm 3^{\circ}$. Agreement of each set of data with a single double sine curve indicates constancy of intensity and gross polarization over an interval of the order of an hour: agreement of the three sets of data shows constancy of the gross polarization over 8 months.

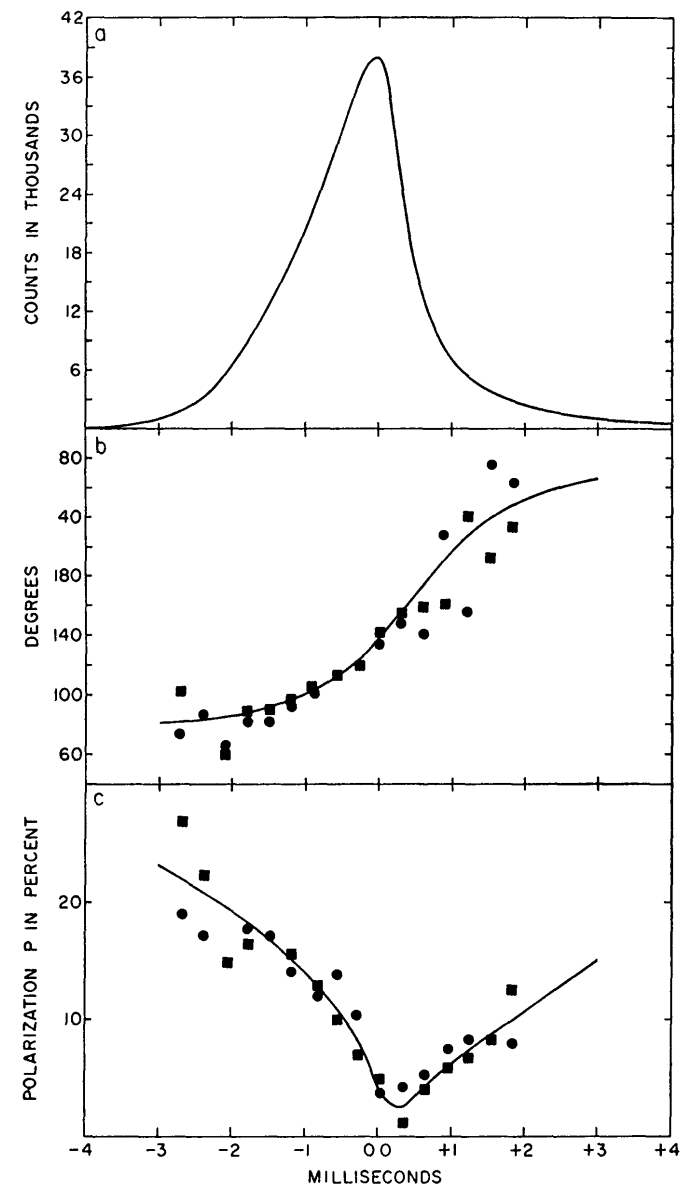

Fig 2

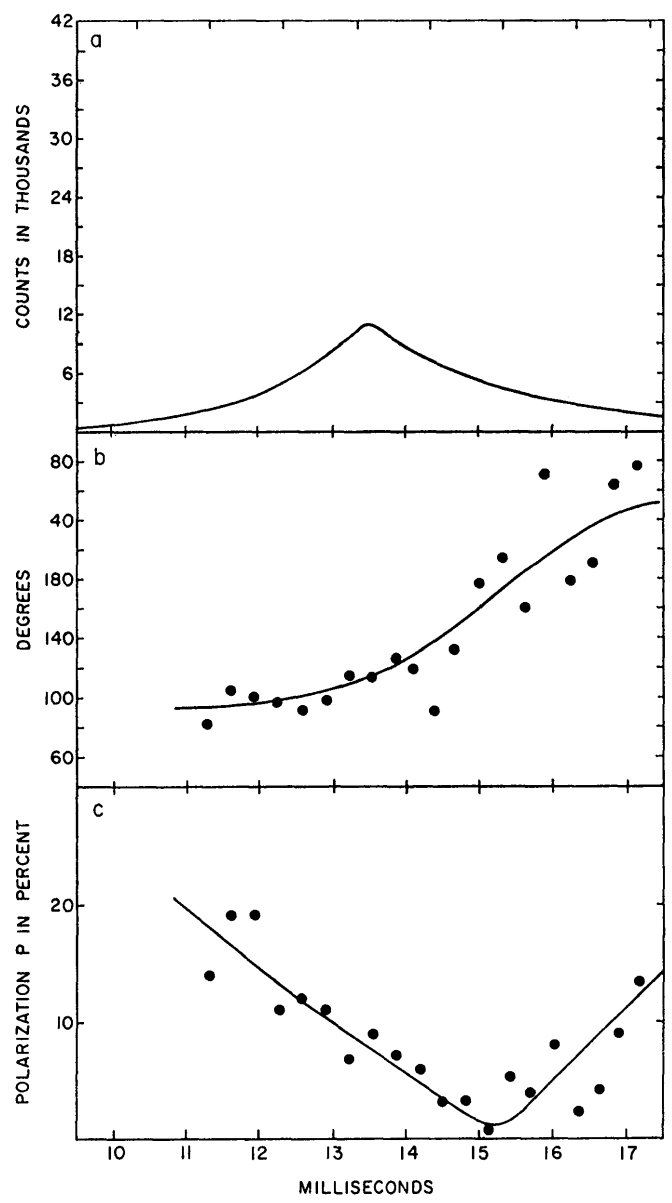

FIG 3

Fig. 2.-Average time changes of linear polarization within the main pulse, for the nights of 1969 August 18 and 19 (average, filled circles) and 1969 October 19 (filled squares). Top panel is a sketch of the averaged light curve (total intensity) through the main pulse. Center panel shows the position angle $\theta$ of the electric vector: the curve is a theoretical one, based on a model discussed in $\S$ IV. Lower panel shows the percent polarization, $P$; curve is an eye estimate. The zero of time is taken to be the peak of the main pulse.

Fig. 3.-Average time changes of linear polarization within the subpulse. Symbols and layout are as in Fig. 2. Note the very large phase shift between the polarization behavior and the total intensity. The zero of time is the peak of the main pulse. 
show the results for the main pulse and subpulse. The curves drawn through the points in Figures $2 b$ and $3 b$ are theoretical, based on a general geometrical model described in $\S$ IV below.

Both $P$ and $\theta$ change through the pulses. In the main pulse, $P$ drops smoothly from about 25 percent in the leading edge to a minimum of about 2 percent just after the peak of the light curve, then increases in a roughly symmetric fashion to at least 10 percent in the trailing edge. The polarization can be followed further into the leading wing than into the trailing wing because of the asymmetric intensity. The polarization behavior of the subpulse is similar to that of the main pulse, even though the subpulse is twice as long as the main pulse and the intensities are asymmetric in opposite senses.

The minimum polarization in the pulses is about 2 percent at an angle $\theta \simeq 160^{\circ}$ with no correction for interstellar polarization. Hiltner (1957) has measured the polarization of two stars in the direction of the Crab Nebula and has found interstellar polarization of a few percent at a position angle of $149^{\circ}$. It is therefore likely that the minimum polarization observed in the pulses is mainly interstellar, and that the minimum intrinsic polarization of the pulses is substantially less than 1 percent.

The polarization minima are not coincident with the peaks of the light curves, but follow them by $0.2 \mathrm{msec}$ in the main pulse and $1.7 \mathrm{msec}$ in the subpulse. The peak of the subpulse follows the peak of the main pulse by 41 percent of the pulsar period, while the polarization minima are separated by 46 percent of the period. The polarization decreases with increasing intensity, in sharp contrast to the radio observations of Vela pulsar (Radhakrishnan et al. 1969; Ekers and Moffet 1969), which is the only other pulsar yet known with high polarization and simple, smooth changes of $P$ and $\theta$ within the pulse.

\section{d) Comparison with Radio Observations}

Measurements of the polarization of the Crab pulsar have been made in the radio region at 408 and $430 \mathrm{MHz}$ (Graham, Lyne, and Smith 1970; Campbell, Heiles, and Rankin 1970). Comparison of the radio and optical data is somewhat complicated by the narrowing of the pulses and the appearance of a precursor to the main pulse at these radiofrequencies. The radio polarization drops from 100 percent toward zero through the 2 -msec duration of the precursor, then increases from zero to 40 percent through the $1-\mathrm{msec}$ duration of the main pulse. The radio polarization of the subpulse is weak and is less than 10 percent at its peak. The optical $P(t)$ cannot be explained as the sum of a component proportional to that seen in the radio and an unpolarized component without highly artificial assumptions regarding the time variation of the unpolarized component, since the main optical pulse, with a very high signal-to-noise ratio, shows no indication of having two components. Further, even with an accuracy of the order of $20^{\circ}$, or 7 times smaller than the rotation observed in visible wavelengths, there is no rotation of the plane of polarization within the pulses in the radio emission. The direction of the plane of polarization for the radio measurements has not yet been determined.

The simultaneity of pulse arrival times in radio and optical wavelengths (Conklin et al. 1969) and the general correspondence of intensity profiles clearly indicate that the radio and optical pulses of the Crab are intimately related. On the other hand, the substantial differences in the detailed profiles and in the polarization, the large intrinsic changes in the radio intensities, and the highly unusual intensity distribution, with separate peaks in the radio and the optical-to-X-ray regions, all point to a radio-emission mechanism which is different in detail from that at higher energies.

\section{e) Comparison with Other Optical Observations}

Polarization observations during the period spanned by our observations have been reported by several groups. The first suggestion of the variation of $P$ and rotation of $\theta$ during the pulses was made by Warner et al. (1969). Their measurements are not of high statistical accuracy, but are in satisfactory agreement with ours. 
Cocke et al. (1969) tentatively confirmed the rotation suggested by Warner et al. (1969) but reported variability of the integrated polarization from night to night, and within a given night on a 10-minute time scale. Such variability would appear as scatter in the points of our Figure 1, and is not confirmed. Later work by Cocke et al. indicates that the apparent variability may have been instrumental in origin (Disney, private communication).

A more detailed study was made by Wampler et al. (1969), with time resolution and accuracy similar to the measurements reported here. However, the intensities observed at repeated Polaroid settings showed fluctuations, which they interpreted as being due to clouds; in attempting to correct for these, they reduced their data in such a way that they are valid only in the wings of the pulses, where the polarization is high. Their procedure was to divide each point in the pulse at each angle by the total area under the main pulse at that angle. The error in the polarization due to this procedure will be of the same order as the integrated polarization ( 7 percent). This effect can in fact be seen directly in their data (their Fig. 4). Instead of dropping to zero (as in our Figs. 2 and 3), their computed polarization levels off at about 7 percent, which is the in tegrated polarization. However, their measurements can be compared with ours in the leading edges of the pulses, where the polarization is large compared with 7 percent, and $P$ and $\theta$ will be changed little. Their data in this part of the light curve are in fact in reasonably good agreement with ours. They also calculated the integrated polarization. Their average value of $6.5 \pm 0.9$ percent at $107^{\circ} \pm 6^{\circ}$ is in surprisingly good agreement with ours, in view of the fluctuations.

\section{SOME IMPLICATIONS OF THE OBSERVATIONS}

The evidence is by now overwhelming that the pulsars are rotating objects, probably neutron stars. The secular constancy and precise phase lock of the intensity and polarization over months leave little doubt that the optical pulses of the Crab pulsar are due to a stable pattern of polarized radiation, fixed in the pulsar, which is being azimuthally scanned as the object rotates. It is probably worth noting that, independently of other considerations, this implies in a straightforward way the existence in the Crab pulsar of a quasi-permanent magnetic field, whose existence has been assumed in much of the theoretical discussion of pulsars: The pulsed nature of the radiation requires localization of the emission, while the polarization requires a fixed local direction, and the most natural way to satisfy both of these requirements is, in one way or another, through the agency of a magnetic field.

In addition to a magnetic field, emission mechanisms which are likely to be considered will also involve high-energy particles, and the observed constancy of the emission and polarization on time scales from tens of milliseconds to a year will require a mechanism which maintains a constant particle flux. The temptation is strong to suppose that the particle flux may itself be generated by the rotating magnetic field.

The rotation of the electric vector within the Crab pulses can be interpreted in terms of a very general model which yields interesting conclusions concerning the geometry of the pulsar and its connection with the surrounding nebula. We have argued above that the observed pulses are produced by a polarized-radiation pattern, fixed in the pulsar, which is scanned as the pulsar rotates. Furthermore, the smoothness of the variations of $P$ and $\theta$ with angular resolution of the order of degrees (time resolution of the order of hundreds of microseconds) suggests that the pattern is relatively simple on this scale. As a first approximation, therefore, we will adopt the following model: We assume that for each of the two pulses there is a unit vector $p$, fixed in and rotating with the pulsar, such that the apparent direction of the electric vector, $\theta$, at any instant is parallel or perpendicular to the projection of $p$ on the plane of the sky. It is supposed that $p$ is associated with a single fixed longitude (in pulsar-centered coordinates) but not necessarily with a single latitude. In particular, if the emission pattern is a fan beam, $p$ will be determined by an average of the emission properties of the beam in latitude. 
A special case of this model has previously been discussed by Wampler et al. (1969) for the optical pulses of the Crab pulsar and by Radhakrishnan et al. (1969) for the radio pulses of the Vela pulsar. The emission pattern was assumed by these authors to be a pencil beam, and $p$ was assumed to be in the direction of the pulsar-centered radius vector of a point on the surface of the pulsar.

For the more general case, the time behavior of $\theta$ is governed by the equation

$$
\cot \theta=\frac{\tan \beta \cos b-\cos (\omega t+\epsilon) \sin b}{\sin (\omega t+\epsilon)},
$$

which is a simple generalization of the equation given by Wampler et al. (1969). Here $\omega$ is the rotation frequency, $b$ is the latitude of the observer with respect to the pulsar, $\beta$ is the angle between $p$ and the equator of the pulsar, and $\epsilon$ is the angle between the projection of $p$ on the pulsar's equator and the meridian plane of the longitude associated with $p$. That is, if $\left(p_{z}, p_{\rho}, p_{\phi}\right)$ are the cylindrical coordinates of $p$ in a frame rotating with the pulsar, then $\tan \beta=p_{z} /\left(p_{\rho}^{2}+p_{\phi}^{2}\right)^{1 / 2}$ and $\tan (\omega t+\epsilon)=p_{\rho} / p_{\phi}$. The phase has been so chosen that, at $t=0$, the observer is at the longitude associated with $p$.

The sweep of $150^{\circ}$ in $\theta$ during only $60^{\circ}$ of the pulsar rotation requires both $\beta$ and $b$ in equation (1) to be small. If one looks at the data in detail and compares them with equation (1), a conservative estimate is that for both pulses, $\beta$ cannot be more than $25^{\circ}$ and $b$ must be within $10^{\circ}$ of $\beta$. Thus the observer must in any case be less than $35^{\circ}$ from the equator of the pulsar, ${ }^{1}$ and, if the emission pattern is a radial pencil beam, the emitting region must lie within $25^{\circ}$ of the equator. It should be noted that the near equality of $b$ and $\beta$ is a necessary but not sufficient condition for the emission pattern to be a pencil beam, and that the same may be said for the radio observations of the Vela pulsar.

The curves drawn in Figures $2 b$ and $3 b$ are computed with the following parameters: $b=7^{\circ}$ (i.e., the observer is almost in the equatorial plane of the pulsar); for the main pulse $\beta=0^{\circ}$ (i.e., $p$ is orthogonal to the rotation axis), and for the subpulse $\beta=6^{\circ}$; the phase difference $\epsilon$ (main) $-\epsilon(\mathrm{sub})$ is $10^{\circ}$. This phase difference is a linear combination of the relative longitudes associated with the vectors $p$ and their angles with respect to the local meridian planes, and the two effects cannot be separated without a more specific model. The differences in the angles are seen in the slightly different shapes for the $\theta(t)$ curves: this is most clearly seen in the leading edges, where the accuracy for both the main pulse and subpulse is relatively high, and shows up as a $10^{\circ}$ vertical difference in the flat parts of the curves.

In the model, the run of $\theta$ with time will show an inversion symmetry about the moment, $\omega t+\epsilon=0$, when $p$ lies in the plane defined by the rotation axis and the direction of the observer, at which time the direction $\theta$ will be either parallel or orthogonal to the projection of the rotation axis on the plane of the sky. Such symmetry is in fact observed in both pulses, and its occurrence corresponds to that of polarization minimum in Figures $2 c$ and $3 c$.

The observed direction of $\theta$ at the center of symmetry is $160^{\circ} \pm 7^{\circ}$, and this angle agrees very well with the angle of polarization in the nebula immediately surrounding the pulsar, which was found to be $159^{\circ} \pm 2^{\circ}$ (see $\S$ III $a$ above). On the assumption that the polarization in the nebula is due to synchrotron radiation, for which the magnetic field is orthogonal to $\theta$, this means that the pulsar's rotation axis is either parallel or orthogonal to the general magnetic field in the immediate vicinity of the pulsar. It should be emphasized that this unexpected result is based on a very general geometrical model, which seems likely to be valid, at least in first order, for any physical model.

The agreement between the direction of the rotation axis and the direction of polariza-

1 That is, the rotation axis of the pulsar must be within $35^{\circ}$ of the plane of the sky; this is consistent with a recent model, relating NP 0531, NP 0527, and I Gem, by Gott, Gunn, and Ostriker (1970). 
tion in the nebula seems too close to be accidental and suggests a direct causal connection between the pulsar and the surrounding field. The implication is strong that the magnetic field, at least in the vicinity of the pulsar, may have been produced by the pulsar itself at some stage in its history. One possibility, for example, is that the magnetic field was spun off the star as it collapsed. It may also be noted that recent analyses of the electromagnetic field around the pulsar, by Goldreich and Julian and by Ostriker and Gunn, indicate that the external field will be directly related to any field at the surface of the pulsar, and that the rotation axis will be an axis of symmetry of the external field. There is a substantial literature concerned with the origin of the ordered magnetic field in the Crab Nebula, and the question is still very much open, but the present data are the first direct observational evidence of a simple connection between the collapsed star and the external field.

We are much indebted to Stephen Paavola and R. Napier for invaluable assistance in obtaining and reducing the data, and to Peter Goldreich, J. P. Ostriker, and A. R. Sandage for helpful comments. One of us (N. V.) would like to express his thanks to Dr. H. W. Babcock for the use of the facilities at the Hale Observatories.

\section{REFERENCES}

Baade, W. 1956, B.A.N., 12, 312.

Boynton, P. E., Groth, E. J., II, Partridge, R. B., and Wilkinson, D. J. 1969, I.A.U. Circ., No. 2179

Campbell, D. B., Heiles, C., and Rankin, J M. 1970, Nature, 225, 527.

Cocke, W. J., Disney, M. J., and Gehrels, T. 1969, Nature, 223, 576.

Conklin, E. K., Howard, H. T., Miller, J. S., and Wampler, E. J. 1969, Nature, 222, 552.

Ekers, R. D., and Moffet, A. T. 1969, A p. J. (Letters), 158, L1.

Freeman, K. C., Rodgers, A. W., Rudge, P. T., and Lyngå, G. 1969, Nature, 222, 459.

Gott, J. R., Gunn, J. E., and Ostriker, J. P. 1970, Ap J. (Letters), 160, L91.

Graham, D. A., Lyne, A. G., and Smith, F. G. 1970, Nature, 225, 526.

Hiltner, W. A. 1957, Ap. J., 125, 300.

Kristian, J. 1970, Pub. A.S.P., 82, 456.

Lynds, C. R., Maran, S. P., and Trumbo, D. E. 1969, A p. J. (Letters), 155, L121.

Miller, J. S., and Wampler, E. J. 1969, Nature, 221, 1037.

Oke, J. B. 1969, Ap.J. (Letters), 156, L49.

Radhakrishnan, V., Cooke, D. J., Komesaroff, M. M., and Morris, D. 1969, Nature, 221, 443.

Sandage, A. R., and Smith, L. 1963, Ap.J., 137, 1057.

Sandage, A. R., and Visvanathan, N. 1969, Ap.J., 157, 1065.

Scargle, J. D. 1969, A p. J., 156, 401.

Seeger, C. L., and Westerhout, G. 1957, B.A.N., 13, 312.

Visvanathan, N. 1967, Ap.J. (Letters), 150, L149.

$$
\text { . 1968, ibid., 153, L19. }
$$
1970, in preparation.

Wampler, E. J., Scargle, J. D., and Miller, J. S. 1969, A p. J. (Letters), 157, L1.

Warner, B., Nather, R. E., and MacFarlane, M. 1969, Nature, 222, 233.

Westphal, J. A., Kristian, J., Snellen, G., Sandage, A. R., Schmidt, M., Oke, J B., Neugebauer, G., and Becklin, E. E. 1969, Ap.J. (Letters), 155, L109. 
